\title{
Synthesis of Aromatic Polyamide-Poly(methyl methacrylate) Graft Copolymers by the Macromonomer Method
}

\author{
Yoshiki CHUJO, ${ }^{*}$ Hisaaki KoBAYASHI, and Yuya YAMASHITA \\ Department of Synthetic Chemistry, Faculty of Engineering, \\ Nagoya University, Furo-cho, Chikusa-ku, Nagoya 464, Japan
}

(Received January 8, 1988)

\begin{abstract}
Aromatic polyamide-poly(methyl methacrylate) graft copolymers were prepared from condensation-type macromonomers. Dicarboxyl-terminated poly(methyl methacrylate) was copolycondensed with $p$-aminobenzoic acid in the presence of $m$-phenylenediamine using triphenylphosphite and pyridine. The resulting graft copolymers were characterized by their ${ }^{1} \mathrm{H}$ NMR and IR spectra. The mechanical properties of poly(methyl methacrylate) or poly(vinyl chloride) films were improved upon addition of small amounts of the resulting graft copolymers.

KEY WORDS Aromatic Polyamide / Graft Copolymer / Macromonomer / Poly(methyl methacrylate) / Poly(vinyl chloride) / Mechanical Properties /
\end{abstract}

Modification of commodity polymers by incorporation of graft copolymers is known to be of considerable importance in various applications. Recently, we have been studying the surface modification of commodity polymers arising from the accumulation of functional segments of tailor-made graft copolymers. ${ }^{1}$ Among the many preparative methods for tailored graft copolymers, the macromonomer technique appears most promising. ${ }^{2}$ Previously, we reported the synthesis of dicarboxyl-terminated poly(methyl methacrylate) as the first example of condensationtype macromonomers. ${ }^{3}$ By using this macromonomer, various kinds of graft copolymers of polyamides ${ }^{4}$ or polyurethanes ${ }^{5}$ were easily prepared.

This paper describes the polycondensation reactions of dicarboxyl-terminated poly(methyl methacrylate) with aromatic comonomers to produce aromatic polyamidepoly(methyl methacrylate) graft copolymers. Wholly aromatic polyamides (aramids) such as Kevlar and Nomex are known to show high tensile strength and modulus. Ogata et al. reported the preparation of graft copolymers consisting of polyacrylate backbones and aramid branches by reactions between two functional polymers. ${ }^{6}$ The resulting graft copolymers showed the mechanical behavior of thermoplastic elastomers depending on the alkyl groups in polyacrylate chains.

Here we wish to prepare the graft copolymers of aramid-poly(methyl methacrylate) by the macromonomer method. It might be expected to improve the mechanical properties of poly(methyl methacrylate) or of poly(vinyl chloride) by means of small amounts of these graft copolymers.

\section{EXPERIMENTAL}

\section{Materials and Instruments}

Methyl methacrylate (MMA) was washed with $5 \%$ aq $\mathrm{NaOH}$ and $5 \%$ aq $\mathrm{NaCl}$, dried over $\mathrm{CaH}_{2}$, and distilled under reduced pressure. 2,2'-Azobisisobutyronitrile (AIBN) was recrystallized from methanol. Thiomalic acid

* Author to whom correspondence should be addressed. Present address: Department of Synthetic Chemistry, Faculty of Engineering, Kyoto University, Yoshida, Sakyo-ku, Kyoto 606, Japan. 
(TMA), p-aminobenzoic acid, triphenylphosphite and lithium chloride were obtained commercially and used without further purification. $m$-Phenylenediamine was distilled under nitrogen atmosphere. All solvents were dried and purified by distillation. Poly(methyl methacrylate) (PMMA) and poly(vinyl chloride) (PVC) were kindly supplied from Mitsubishi Rayon Co., Ltd., and Toa Gosei Kagaku Kogyo Co., Ltd., respectively and used after reprecipitation and drying.

IR and ${ }^{1} \mathrm{H}$ NMR spectra were obtained with a JASCO IRA-1, and a JEOL JNM PMX-60 $(60 \mathrm{MHz})$, respectively. GPC was carried out on a Toyo-Soda HLC-802 UR after calibration with standard polystyrene samples. Molecular weights were determined by VPO in benzene using a Hitachi-115 instrument.

\section{Synthesis of Macromonomers}

In a $50 \mathrm{ml}$ glass ampoule, MMA $(8.22 \mathrm{~g}$, $82.2 \mathrm{mmol})$, TMA $(0.36 \mathrm{~g}, 2.43 \mathrm{mmol})$, AIBN $(0.12 \mathrm{~g}, \quad 0.75 \mathrm{mmol})$ and tetrahydrofuran (THF) $(20 \mathrm{ml})$ were placed and sealed under vacuum. Polymerization was carried out at $60^{\circ} \mathrm{C}$ for $3 \mathrm{~h}$. The resulting macromonomer was precipitated into petroleum ether, purified twice by reprecipitation from benzene into petroleum ether, once from acetone into water, then dried under vacuum. The carboxyl-group content was determined by titrating the polymer in THF with $0.02 \mathrm{~N}$ aq $\mathrm{KOH}$, using phenolphthalein as an indicator.

\section{Synthesis of Graft Copolymer}

Under nitrogen, a mixture of macromonomer $(5.55 \mathrm{~g}, 0.49 \mathrm{mmol}), m$-phenylenediamine $(0.053 \mathrm{~g}, \quad 0.49 \mathrm{mmol}), \quad p$-aminobenzoic acid $(0.618 \mathrm{~g}, \quad 4.51 \mathrm{mmol})$, triphenylphosphite $(1.55 \mathrm{~g}, 5.00 \mathrm{mmol}), \mathrm{LiCl}(0.09 \mathrm{~g}, 2.12 \mathrm{mmol})$, pyridine $(6 \mathrm{ml})$ and $N$-methyl-2-pyrrolidone (NMP) $(24 \mathrm{ml})$ was placed in a $200 \mathrm{ml}$ flask equipped with a reflux condenser and heated to $100^{\circ} \mathrm{C}$ for $4 \mathrm{~h}$. The resulting reaction mixture was poured into methanol. The polymer was obtained almost quantitatively and purified by reprecipitation from dichloroacetic acid into water-methanol $(1: 1, \mathrm{v} / \mathrm{v})$ and drying in vacuo. The viscosities were measured in conc. sulfuric acid at $30^{\circ} \mathrm{C}\left(c=0.5 \mathrm{~g} \mathrm{dl}^{-1}\right)$.

\section{Formation of Films}

A mixture of graft copolymer and PMMA $\left(\bar{M}_{n}=52400\right)$ was suspended in NMP and poured into a mixture of methanol and petroleum ether. After Soxhlet extraction with diethyl ether and drying in vacuo, the polymer blend was heated to $200^{\circ} \mathrm{C}$ under pressure between Teflon plates. PVC $\left(\bar{M}_{n}=62400\right)$ films containing graft copolymers were prepared in a similar way.

\section{RESULTS AND DISCUSSION}

The synthesis of graft copolymers of aramid-PMMA was carried out according to Scheme 1. The dicarboxyl-terminated macromonomer (1) was prepared by free radical polymerization of methyl methacrylate initiated by AIBN in the presence of thiomalic acid (TMA) as a chain transfer agent as reported previously. ${ }^{3,4}$ In this reaction, the molecular weight of 1 was easily controlled by changing the feed ratio of chain transfer agent (TMA) to monomer (MMA) for low conversions. The terminal dicarboxyl group was effectively introduced at one end of the macromonomer chain.

As shown in Scheme 1, 1 was copolymerized with $p$-aminobenzoic acid in the presence of $m$ phenylenediamine using the triphenylphosphite-pyridine system. ${ }^{7}$ The results of the preparation of graft copolymers (2) are summarized in Table I. 2 was found to be insoluble in common organic solvents. Even PMMA-rich graft copolymers (runs 2 or 3 in Table I) were soluble only in trifluoroacetic acid or in sulfuric acid.

The IR spectrum of the resulting graft copolymer (2) is illustrated in Figure 1C together with those for poly ( $p$-aminobenzoic acid) (1A) and PMMA macromonomer (1B) for com- 


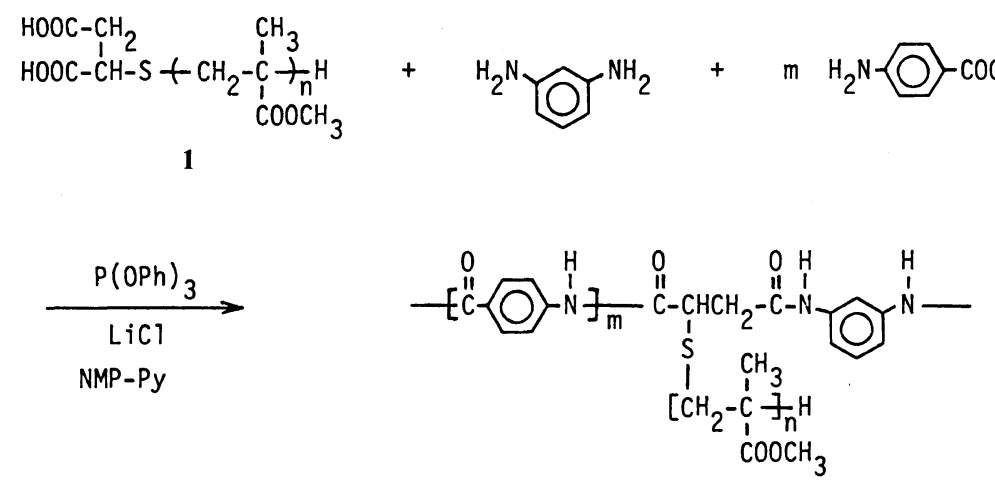

2

Scheme 1.

Table I. Preparation of graft copolymers (2)

\begin{tabular}{|c|c|c|c|c|c|c|}
\hline \multirow{3}{*}{ Run } & \multicolumn{3}{|c|}{ Feed } & \multicolumn{3}{|c|}{ Graft copolymer } \\
\hline & PM & MA & MMA & PMMA & MMA & \\
\hline & $\bar{M}_{n}^{\mathrm{a}}$ & $\mathrm{mol} \% \mathrm{~b}$ & $w t^{\circ} \%$ & $\mathrm{~mol} \%^{\mathrm{c}}$ & $w t^{\circ}{ }^{c}$ & \\
\hline 1 & 7450 & 1.20 & 42.2 & 1.05 & 39.2 & 1.15 \\
\hline 2 & 11300 & 4.76 & 81.2 & 4.00 & 78.9 & 0.53 \\
\hline 3 & 11300 & 9.82 & 89.6 & 8.40 & 88.8 & 0.40 \\
\hline
\end{tabular}

a Calculated by end-dicarboxyl group titration.

b $1 /(m+1) \times 100$.

c Calculated by $\mathrm{N} \%$ in elemental analyses.

d Measured in conc $\mathrm{H}_{2} \mathrm{SO}_{4}$ at $30^{\circ} \mathrm{C}\left(c=0.5 \mathrm{~g} \mathrm{dl}^{-1}\right)$.

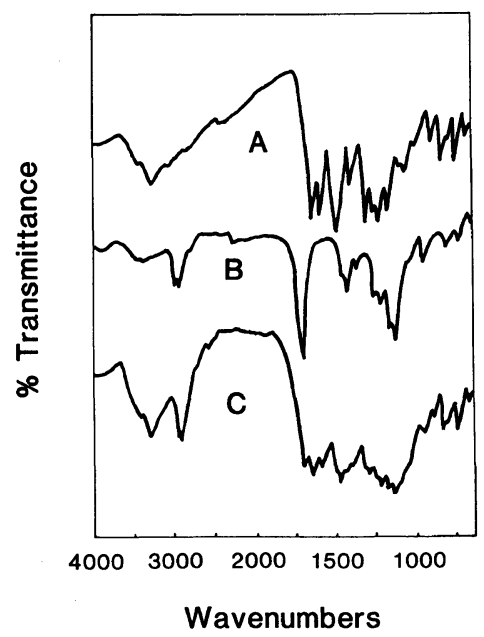

Figure 1. IR spectra of (A) poly( $p$-aminobenzoic acid), (B) poly(methyl methacrylate) macromonomer, and $(\mathrm{C})$ graft copolymer.

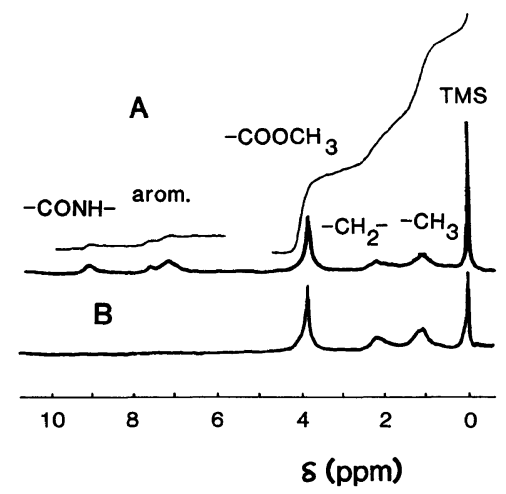

Figure 2. ${ }^{1} \mathrm{H}$ NMR spectra of (A) graft copolymer (run 3 in Table I) in $\mathrm{D}_{2} \mathrm{SO}_{4}$ and (B) (run 1) in $\mathrm{CF}_{3} \mathrm{COOD}$.

parison. The results support the composition of 2.

Figure 2A shows the ${ }^{1} \mathrm{H}$ NMR spectrum of 2 (run 3 in Table I) using sulfuric acid- $d_{2}$ as a solvent. This spectrum shows the structure of aramid and PMMA segments in 2. On the other hand, the graft copolymer with lower MMA content (run 1 in Table I) shows only PMMA segments in the ${ }^{1} \mathrm{H}$ NMR spectrum in trifluoroacetic acid- $d_{1}$ as shown in Figure 2B. Thus, the aramid backbone is relatively insoluble and immobilized compared with the PMMA branches in trifluoroacetic acid. As a result, the peaks of aromatic protons in the aramid segments were hardly seen in its ${ }^{1} \mathrm{H}$ NMR spectrum. The same type of behavior 
was observed in our study on the graft copolymers of PMMA-poly(dimethylethylammoniumethyl methacrylate), which formed a kind of polymeric micelle in an acetonewater mixed solvent. ${ }^{8}$ Thus, the $\mathrm{wt}^{\mathrm{o}} \%$ of MMA in 2 had to be calculated from elemental analysis. Only in the case of run 3 in Table I, the $\mathrm{wt} \%$ of MMA could also be calculated from the integral ratio of the aromatic protons $(\delta 6.9-7.8 \mathrm{ppm})$ to the $-\mathrm{OCH}_{3}$ protons $(\delta 3.8$ ppm) in the ${ }^{1} \mathrm{H}$ NMR spectrum, and was 90.1 $\mathrm{wt} \%$, a value very close to $88.8 \mathrm{wt} \%$ determined by elemental analysis. A slight decrease in the $\mathrm{wt} \%$ of MMA in copolymers was observed compared with that of the feed. This might be explained by assuming that reprecipitation and washing removed some low molecular weight aramids, which were appreciably grafted with PMMA.

The reduced viscosities of 2 , measured in sulfuric acid, are shown in Table I. The graft copolymers were stable in sulfuric acid without change of values of viscosities for several weeks. The viscosities decreased with increasing MMA content. However, even in the case of run 3 , the value of viscosity was higher than 0.4 , indicating that a high molecular weight graft copolymer was obtained by this method.

The graft copolymer (2) was blended with commodity polymers and the mechanical

Table II. Mechanical properties of PMMA films

\begin{tabular}{|c|c|c|c|c|c|}
\hline \multirow{3}{*}{ Run } & \multicolumn{2}{|c|}{ Graft copolymer } & \multirow{3}{*}{$\frac{\mathrm{PABA}^{\mathrm{b}}}{w \mathrm{t}^{\mathrm{o}} \%}$} & \multirow{3}{*}{$\frac{\begin{array}{c}\text { Tensile } \\
\text { modulus }\end{array}}{\mathrm{MPa}}$} & \multirow{3}{*}{$\frac{\begin{array}{c}\text { Ultimate } \\
\text { elongation }\end{array}}{\%}$} \\
\hline & \multirow{2}{*}{$\frac{\text { MMA }}{w t^{\circ} \%}$} & \multirow{2}{*}{$\frac{\text { Feed }^{\mathrm{a}}}{\mathrm{wt} \%}$} & & & \\
\hline & & & & & \\
\hline 1 & - & - & $0^{\mathrm{c}}$ & 1820 & 5 \\
\hline 2 & 88.8 & 5.0 & 0.56 & 1960 & 4 \\
\hline 3 & 78.9 & 5.0 & 1.06 & 2280 & 3 \\
\hline 4 & 72.0 & 5.0 & 1.40 & 1990 & 4 \\
\hline 5 & 78.9 & 10.0 & 2.11 & 2300 & 3 \\
\hline 6 & 39.2 & 5.0 & 3.04 & 1920 & 4 \\
\hline
\end{tabular}

a Amounts of graft copolymers incorporated in films.

b $w t \%$ of the aromatic polyamide segment in blends of PMMA and graft copolymer.

c PMMA alone. properties of the resulting films were examined. PMMA films containing various amounts of 2 (runs 2 and 3 in Table I) were formed by heat pressing between Teflon films. In the case of aramid-rich graft copolymer (run 1), phase separation was observed after blending with PMMA and the resulting films were turbid even for low content of $\mathbf{2}$. By using PMMA-rich graft copolymers (runs 2 and 3), the graft copolymer and PMMA were compatible up to $10 \mathrm{wt} \%$ of 2 and transparent films were obtained. Similarly, transpar-

Table III. Mechanical properties of PVC films

\begin{tabular}{|c|c|c|c|c|c|}
\hline \multirow{3}{*}{ Run } & \multicolumn{2}{|c|}{ Graft copolymer } & \multirow{3}{*}{$\frac{\mathrm{PABA}^{\mathrm{b}}}{w \mathrm{t}^{\mathrm{o}} \%}$} & \multirow{3}{*}{$\frac{\begin{array}{c}\text { Tensile } \\
\text { modulus }\end{array}}{\mathrm{MPa}}$} & \multirow{3}{*}{$\frac{\begin{array}{c}\text { Ultimate } \\
\text { elongation }\end{array}}{\%}$} \\
\hline & \multirow{2}{*}{$\frac{\text { MMA }}{w t^{\circ} \%}$} & \multirow{2}{*}{$\frac{\text { Feed }^{\mathrm{a}}}{w \mathrm{t}^{\mathrm{o}} \%}$} & & & \\
\hline & & & & & \\
\hline 1 & - & - & $0^{\mathrm{c}}$ & 1450 & 200 \\
\hline 2 & 78.9 & 2.0 & 0.42 & 1720 & 50 \\
\hline 3 & 88.8 & 5.0 & 0.56 & 1820 & 20 \\
\hline 4 & 78.9 & 5.0 & 1.06 & 1820 & 32 \\
\hline 5 & 78.9 & 10.0 & 2.11 & 1880 & 17 \\
\hline 6 & 39.2 & 5.0 & 3.04 & 1820 & 16 \\
\hline
\end{tabular}

a Amounts of graft copolymers incorporated in films.

b $\mathrm{wt}^{\%} \%$ of the aromatic polyamide segment in blends of PVC and graft copolymer.

c PVC alone.

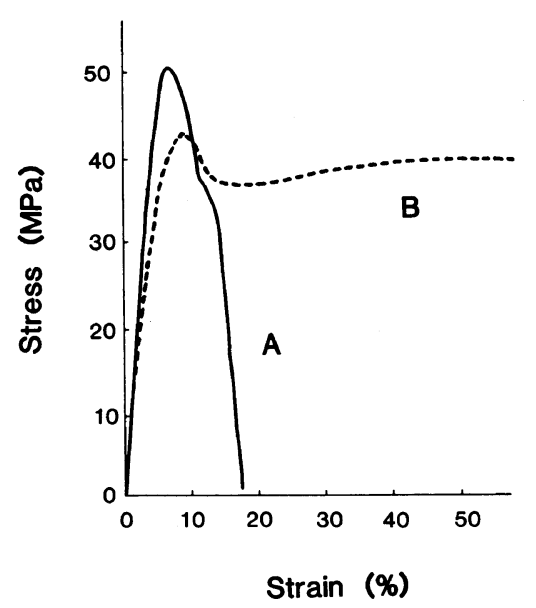

Figure 3. Stress-strain curves of (A) PVC containing graft copolymer (run 5 in Table III) and (B) PVC alone (run 1) at $23^{\circ} \mathrm{C}$ and $\mathrm{RH}=50 \%$ (strain rate $=7.5 \%$ $\min ^{-1}$ ). 
ent PVC films containing various amounts of PMMA-rich graft copolymers were prepared by heat pressing. Tables II and III summarize the mechanical properties, tensile modulus and ultimate elongation, of the resulting PMMA and PVC films, respectively. Aramids are known to show high tensile strength and modulus. As shown in Tables II and III, the tensile modulus slightly increased in both cases of PMMA and PVC upon adding graft copolymer. This property is also shown in Figure 3, which illustrates stress-strain curves of homo-PVC and of PVC containing graft copolymer. However, PVC containing graft copolymers exhibits an ultimate elongation much lower than for PVC alone. The property of low ultimate elongation is considered to be due to the rigid and highly oriented molecular structure of an aramid segment even in the case of low content of graft copolymer.
Acknowledgement. The authors are indebted to Mr. K. Chiba, Toray Co., Ltd. for the examination of mechanical properties of PMMA and PVC films.

\section{REFERENCES}

1. Y. Yamashita, Appl. Polym. Symp., 36, 193 (1981).

2. P. F. Rempp and E. Franta, Adv. Polym. Sci., 58, 1 (1984).

3. Y. Yamashita, Y. Chujo, H. Kobayashi and Y. Kawakami, Polym. Bull., 5, 361 (1981).

4. Y. Chujo, H. Kobayashi, and Y. Yamashita, Polym. Commun., 25, 278 (1984).

5. Y. Chujo, T. Tatsuda, and Y. Yamashita, Polym. Bull., 8, 239 (1982).

6. N. Ogata, K. Sanui, M. Watanabe, and I. Yahagi, $J$. Polym. Sci., Polym. Lett. Ed., 23, 349 (1985).

7. F. Higashi, M. Goto, and H. Kakinoki, J. Polym. Sci., Polym. Chem. Ed., 18, 1711 (1980).

8. Y. Chujo, T. Shishino, Y. Tsukahara, and Y. Yamashita, Polym. J., 17, 133 (1985). 